\title{
Kernos
}

Revue internationale et pluridisciplinaire de religion grecque antique

$20 \mid 2007$

Varia

\section{PACHE Corinne Ondine, Baby and Child Heroes in Ancient Greece}

\section{Véronique Dasen}

URL : https://journals.openedition.org/kernos/340

DOI : $10.4000 /$ kernos.340

ISSN : 2034-7871

\section{Éditeur}

Centre international d'étude de la religion grecque antique

\section{Édition imprimée}

Date de publication : 1 janvier 2007

Pagination : 409-412

ISSN : 0776-3824

\section{Référence électronique}

Véronique Dasen, « PACHE Corinne Ondine, Baby and Child Heroes in Ancient Greece», Kernos [En ligne], 20 | 2007, mis en ligne le 17 mars 2011, consulté le 08 septembre 2022. URL : http:// journals.openedition.org/kernos/340 ; DOI : https://doi.org/10.4000/kernos.340 
dence. De telles lectures pourraient relever d'aspirations néo-mystiques dont C.C. dénonce le caractère syncrétique et bricolé dans une société moderne hypermédiatisée. C'est le quatrième paradigme mis en cause. La réponse à la question du statut orphique de ces textes est négative, dans la mesure où, pour C.C., les graffiti d'Olbia ne sont pas pertinents dans ce contexte, pas plus que l'iconographie des vases apuliens ou le papyrus de Gurôb habituellement appelés à la barre pour soutenir cette lecture orphique. Les défunts accompagnés par les lamelles ont dû se soumettre de leur vivant à des cérémonies initiatiques du type de celle d'Éleusis (ce qui impliquerait des cultes publics), mais avec Dionysos comme dieu principal. Il n'est question ni de transmigration des âmes ni de réincarnation «orphique », mais d'un parcours rituel ponctué d'indications d'ordre pratique fondant un avenir glorieux à accomplir dans l'éternité, dont le modèle est héroïque.

Bon nombre des positions adoptées par C.C. vont à contre-courant d'une certaine doxa, ce dont il est parfaitement conscient. Reconnaissons toutefois que l'armature théorique de cet ouvrage et l'exigence critique dont elle témoigne en décourageront sans doute plus d'un. La doxa a encore de beaux jours devant elle, mais tout historien de la religion grecque qui aborde la question du «mythe», des «rites de passage » ou de l'« orphisme» doit tenir compte des propositions de C.C. qui a, en outre, le grand mérite de tisser des liens entre antiquité et $\mathrm{XXI}^{\mathrm{e}}$ siècle, comme le montre encore la conclusion du livre.

Vinciane Pirenne-Delforge (FNRS - Université de Liège)

PACHE Corinne Ondine, Baby and Child Heroes in Ancient Greece, Urbana / Chicago, University of Illinois Press, 2004. 1 vol., 15,5 × 23,5 cm, X+234 p. (Traditions). ISBN: 0-252-02929-1.

Le monde des héros comporte une catégorie à part, celle des enfants prépubères disparus prématurément de mort violente, volontaire ou accidentelle, avant d'avoir pu se distinguer par de hauts-faits. Leur mort est pourtant expiée par leur héroïsation caractérisée par l'instauration d'un culte souvent lié à la fondation de jeux funéraires. L'oubli dans lequel la recherche les a longtemps tenus est maintenant réparé. Dans cet ouvrage, issu de sa thèse de doctorat à l'Université de Harvard, Corinne Ondine Pache dresse un inventaire détaillé des sources littéraires, iconographiques et archéologiques en s'interrogeant sur les motifs du statut particulier de ces enfants dans l'imaginaire collectif. Les documents rassemblés sont répartis sur un arc chronologique très large, d'Homère à l'époque romaine.

On pourrait inscrire ce travail dans la mouvance d'une double tendance de la recherche actuelle. D'un côté, il participe à l'approfondissement de nos connaissances sur la place de l'enfant dans la société antique, possible uniquement grâce à une approche interdisciplinaire. De l'autre, il explore un champ relativement nouveau, celui de l'histoire des émotions et des sentiments privés qui ne sont pas directement accessibles dans les sources traditionnelles, mais que l'on peut tenter de lire en creux au travers des mythes et des pratiques rituelles.

L'ouvrage se compose de six chapitres consacrés aux enfants de Médée et d'Héraclès, à Linos et Démophon, Pélops, Opheltès-Archémoros ainsi qu’à Mélicerte-Palaemon, comparés aux destins contrastés d'autres enfants mythiques qui ne deviennent pas des héros, tels le petit Glaucos, le fils de Minos qui se noie dans une jarre de miel puis ressuscite, ou les enfants de Niobé, ensevelis à Thèbes, mais sans faire l'objet d'un culte.

L'introduction retrace l'historique des recherches antérieures sur les cultes hérö̈ques en relevant la nécessité de croiser des sources de nature variée pour saisir la nature et le rôle des enfants où l'A. englobe tous ceux qui appartiennent à la classe d'âge désignée par les termes paidion ou pais. 
Les deux premiers chapitres concernent les enfants de Médée et d'Héraclés, tués par leurs propres parents. Chez Euripide, leur mort est une vengeance préméditée par Médée pour détruire Jason; dans d'autres versions du mythe, elle n'est cependant pas directement responsable: les enfants sont victimes de la colère des Corinthiens qui violent l'asile du sanctuaire d'Héra où Médée aurait caché ses fils avant de s'enfuir. Comment expliquer l'extraordinaire popularité de ce mythe, dont témoignent la richesse et la longue durée de son iconographie ? L'A. ne propose pas de réponse, mais évoque le succès de ses versions théâtrales. Cette explication ne convainc qu'à moitié et doit être complétée par des réflexions d'ordre anthropologique : la fortune du récit est lié à l'importance culturelle du motif de la maternité inversée, omniprésente dans les histoires grecques de démons croquemitaines, toujours féminins (Gellô, Mormô...), qui traduisent le pouvoir occulte et inquiétant des femmes sur la fécondité, ici amplifié par la dimension de sorcière de Médée, ailleurs transposé sur la figure de la vieille femme stérile transformée en tueuse de nouveaunés ( $c$. S.I. Johnston, "Defining the dreadful: remarks on the Greek child-killing demon », in M. Meyer, P. Mirecki (éds), Ancient Magic and Ritual Power, Leiden, 1995, p. 361-387). Ces figures maléfiques, humaines ou divines, déchargent les vivants de la culpabilité des morts périnatales, maternelles et infantiles. Cette facette échappe complètement au personnage d'Héraclès dont la folie meurtrière est loin d'égaler la popularité des méfaits de Médée. La menace sur la fécondité que représente Médée finit par se projeter sur toute la communauté des Corinthiens. Dans le récit de Pausanias (II, 3, 7), le meurtre injuste des enfants de la magicienne cause un fléau qui frappe de mort les petits enfants de Corinthe. Il n'est apaisé que par l'instauration d'un culte expiatoire accompli par 7 garçons et 7 filles reclus dans le sanctuaire pendant un an, vêtus d'habits de deuil ( $c$. les enfants lapidés d'Artémis Kondylea; Pausanias, VIII, 23, 6-7). Toutefois, ce genre de raisonnement échappe à l'A., qui n'est guère familiarisée avec les acquis récents de l'anthropologie culturelle.

Le $3^{e}$ chapitre est consacré aux destins de Linos et Démophon, connus uniquement par des sources littéraires. Dans les deux cas, les enfants sont héroïsés suite à l'intervention directe d'une divinité. Enceinte des œuvres d'Apollon, Psamathé, la fille du roi Krotopos d'Argos, accouche d'un fils, Linos, qu'elle abandonne dans la campagne où un berger le recueille. Le bambin est bientôt dévoré par les chiens du roi et Psamathé mise à mort par son père. Ce récit dénonce-t-il, comme l'A. le suggère, le comportement dangereux d'une mère, qui met involontairement en péril la survie de son petit, à l'instar de Médée qui croit protéger ses enfants en les cachant dans le temple d'Héra? L'idée parait incongrue. Le récit me semble plutôt renvoyer à deux topos bien connus : la mort atroce du petit Linos, déchiré par des chiens bergers, évoque en creux les nombreux récits qui montrent à l'inverse un bébé miraculeusement sauvé par des animaux (cf. G. Binder, Die Aussetzung des Königskindes. Kyros und Romulus, Meisenheim am Glan, 1964; D. Briquel, «Les jumeaux à la louve et les jumeaux à la chèvre, à la jument, à la chienne, à la vache », in R. Bloch (éd.), Recherches sur les religions de l'Italie antique, Genève, 1976, p. 73-97). Ce motif s'articule au sort de Psamathé qui se réfère à un autre topos, celui de la parthénos séduite qui doit accoucher secrètement en abandonnant son enfant, et subit un lourd châtiment pour avoir transgressé les règles sociales ( $c f$. G. Sissa, Le corps virginal. La virginité féminine en Grèce ancienne, Paris, 1987, p. 110116). Le destin tragique de Psamathé et de Linos parle ainsi également de l'impuissance des femmes face à la violence masculine, incarnée tant par le roi que par ses chiens. Comme dans l'histoire de Médée, la mort de Linos entraîne un fléau envoyé par Apollon qui extermine les enfants de la région. À nouveau, seule l'institution d'un culte permet de restaurer l'ordre dans la cité. Linos reçoit un culte expiatoire à Argos où son sort - et celui de sa mère - sont pleurés. L'A. souligne que les textes parlent de chiens sacrifiés en nombre, un détail que relèveront les archéologues qui observent régulièrement la présence de chiens dans ou à proximité de tombes d'enfants, en Grèce comme à Rome. 
Le sort de Démophon, que Déméter tente en vain d'immortaliser par le feu, est d'un autre ordre. Metaneira prend peur devant les agissements de la déesse qui, comme Thétis, abandonne l'enfant à son sort de mortel. Dans certaines versions du mythe, Démophon meurt aussitôt et des jeux funéraires sont institués à Éleusis. Pour l'A., le récit sert à dire que la peur maternelle représente une force destructrice qui cause la mort des enfants (p. 83). Une autre interprétation est possible. Le récit confronte deux femmes qui veulent la survie du bébé, une déesse qui connait les procédés d'immortalisation et une mère vigilante, comme toutes les mères mortelles. L'issue dramatique de l'histoire montre que l'immortalisation terrestre est un rêve inaccessible et que les procédés divins échappent à la compréhension des humains qui doivent assumer leur finitude, tel Achille abandonné par Thétis. Il faut également souligner que le contexte de l'épisode de Démophon dans l'Hymne homérique à Déméter est probablement étiologique et que le souci de Déméter pour le petit est une sorte de préfiguration mythique de la sollicitude dont ses mystères témoignent envers les humains mortels. Le cas liminal de Pélops, qui ne meurt pas mais ressuscite, est intégré par l'A. dans le schéma des enfants tués par leurs parents, ici Tantale, et associé plus tard à l'institution des jeux athlétiques d'Olympie.

La $2^{e}$ moitié de l'ouvrage est occupée par les personnages d'Opheltès-Archémoros et Mélicerte-Palaemon, bien documentés du point de vue littéraire, iconographique et archéologique. En commun, les enfants changent d'identité après leur mort dont l'expiation est à nouveau à l'origine de jeux funéraires athlétiques, ici les jeux Néméens et Isthmiques. L'inadvertance d'Hypsipyle est la cause de la mort du petit Opheltès qu'on lui a confié. Pour s'occuper de la troupe des Sept qui recherche une source pour se désaltérer, la nourrice dépose dans l'herbe l'enfant qui s'endort et se fait mordre par un serpent. Avec pertinence, l'A. note les rapports ambivalents du sommeil et de la mort, et associe la variante qui décrit l'enfant cueillant des fleurs à l'image de Coré-Perséphone. Ces thèmes se retrouvent dans les berceuses grecques dont l'A. nous offre une recension bienvenue. La découverte à Némée d'un herôon du VI ${ }^{\mathrm{e}}$ siècle avec des figurines enfantines en terre cuite et en bronze semblent confirmer l'importance de cette figure fondatrice juvénile. Mélicerte-Palaemon connait un autre destin. Sa mère Ino se jette à la mer avec lui, un culte et des jeux sont institués à l'Isthme, là où un dauphin ramène son corps.

De nombreux points communs relient ces récits : l'institution de cultes à connotation chthonienne (la réclusion, les habits noirs et les cheveux coupés des enfants de Corinthe, les cérémonies nocturnes en l'honneur des enfants d'Héraclès, les rites secrets dans le sanctuaire de Palaemon), l'exécution de chants funéraires (le «linos» d'Argos), ainsi que la fondation de jeux athlétiques avec une dimension initiatique (Éleusis, Thèbes, Olympie, Némée, Isthme).

L'ouvrage se termine par une conclusion très succincte. Toutes les observations récoltées auraient pu être commentées dans une discussion générale plus étoffée, situant par exemple les résultats dans une perspective comparatiste, et en proposant des pistes. S'il est clair que dans une société qui valorise le masculin, presque tous ces enfants sont des garçons, où sont les filles? Leur traitement différencié (p. 63) aurait mérité un développement particulier, de même que les brèves réflexions sur les responsabilités maternelles et paternelles. On peut souscrire à la thèse principale de l'A. qui voit dans ces récits une réponse au chagrin, voire au sentiment de culpabilité des parents, une manière de donner un sens à la mort prématurée et de faire acte de mémoire. Il faudrait aussi les lire à la lumière de la place ambivalente de la maternité dans la cité grecque, à la fois indispensable et inquiétante (voir en dernier lieu L. Bodiou, P. Brulé, L. Pierini, «En Grèce antique, la douloureuse obligation de la maternité », Clio, Histoire, Femmes et Societés 21 [2005], p. 17-42). Ces réflexions auraient aussi pu être développées en les reliant aux travaux de M. Golden sur 
l'impact de la forte mortalité infantile sur «le sentiment de l'enfance» (p. ex. «Did the ancients care when their children died? », G\&R 35 [1988], p. 152-163). Le personnage de Médée, comme ceux d'autres figures effrayantes, constitue l'autre face du même souci parental et familial, et il serait très intéressant de retracer dans les récits tous les motifs d'inversion dont ils sont tissés (l'enfant trouvé dévoré par des chiens bergers, la mère tueuse...). Assorti d'un index et d'une bibliographie, l'ouvrage est de facture très soignée, le choix éditorial de souligner d'un double trait les mots des citations lui donnant toutefois un graphisme un peu scolaire.

Véronique Dasen

(Université de Fribourg)

BitTrich Ursula, Aphrodite und Eros in der antiken Tragödie. Mit Ausblicken auf motivgeschichtlich verwandte Dichtungen, Berlin / New York, Walter de Gruyter, 2005. 1 vol. $16 \times 23 \mathrm{~cm}$, VIII +218 p. (Untersuchungen zur antiken Literatur und Geschichte, 75). ISBN : 3-11-0188555-5.

Cet ouvrage est le fruit d'une dissertation présentée à la Rheinischen Friedrich-WilhelmsUniversität de Bonn en 2004-2005 et propose une approche strictement littéraire du thème. Il est important de souligner d'emblée cette caractéristique de l'ouvrage, afin que le lecteur n'aille pas y chercher ce que l'A. n'a pas voulu livrer, en l'occurrence une perspective religieuse ou plus largement anthropologique. De ce point de vue, un tel livre est aux antipodes de L'Éros dans la Grèce antique de Claude Calame (Paris, Belin, 1996; trad. angl. Princeton UP, 1999), ce qui ne suffit pourtant pas à expliquer l'absence d'un tel livre de l'horizon historiographique de cette thèse allemande. Quoi qu'il en soit, l'ouvrage adopte un plan chronologique, qui va d'une introduction sur les textes archaïques ("Vor der Tragödie», mais les auteurs lyriques sont presque totalement absents) jusqu'à Nonnos de Panopolis, en consacrant le cœur du propos aux auteurs tragiques, puis à d'autres auteurs grecs mêlés d'auteurs latins. Le moteur de l'exposé consiste à suivre l'image d'Aphrodite et d'Éros à travers les œuvres littéraires anciennes, à mettre l'accent sur certains topoi les concernant, et à montrer comment, de dieux qu'ils étaient, ils se transforment de plus en plus en figures à la fois rhétoriques et psychologiques. Cette évolution serait liée au fait que ces deux divinités sont traversées par la tension et l'oscillation entre action cosmique et action sur l'individu. De là proviendraient, par exemple, l'ambivalence d'Aphrodite et de son pouvoir, ou bien la figure double d'Éros. Ce dernier est aussi un dieu de la colère et de la vengeance (ultor), puis un rebelle dompté, enfin le dieu de l'amour réciproque.

Le premier grand thème que traite le livre est la puissance de la déesse et l'invincibilité d'Éros. Aphrodite ne se laisse saisir que dans la tension entre une divinité puissante et une forme rhétorique par laquelle les hommes justifient leur faiblesse. Les fragments des tragiques porteraient les signes de cette déesse au pouvoir cosmique, qui semble se placer sur le même plan hiérarchique que Zeus. Éros est son fils et son serviteur. Ces mêmes tragiques développent le thème de l'amour-nosos provoqué par la déesse. Le thème du nosos montrerait bien, selon l'A., que le coéfficient humain commence à s'imposer dans la représentation de la déesse. Dans les tragédies d'Euripide, et surtout dans la trilogie troyenne, l'individu « démythologise » la passion qui prend davantage place dans l'intériorité, ce qui n'implique pas nécessairement que l'auteur tragique partage point par point la posture de ses personnages. Dans l'Alexandros, Aphrodite joue un rôle important et une correspondance s'instaure entre le cadre mythologique et l'action des personnages. Dans la Phèdre de Sénèque, Vénus est bien plus marginale qu'Aphrodite chez Euripide. Le thème du furor correspond à celui du nosos, mais la malédiction de la déesse ne constitue plus que le cadre extérieur de l'action. 